Research Article

\title{
Design and Verification of Reactor Power Control Based on Stepped Dynamic Matrix Controller
}

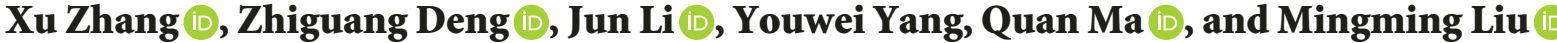

Nuclear Power Institute of China, Science and Technology on Reactor System Design Technology Laboratory, Chengdu 610213, China

Correspondence should be addressed to Quan Ma; maquannpic@163.com

Received 3 July 2019; Revised 12 September 2019; Accepted 24 September 2019; Published 3 November 2019

Academic Editor: Eugenijus Ušpuras

Copyright (c) $2019 \mathrm{Xu}$ Zhang et al. This is an open access article distributed under the Creative Commons Attribution License, which permits unrestricted use, distribution, and reproduction in any medium, provided the original work is properly cited.

As key equipment in nuclear power plant, the reactor power control system is adopted to strictly control and regulate the reactor power of a PWR (pressurized water reactor) in a nuclear power plant. A well-optimized predictive control algorithm based on SDMC (stepped dynamic matrix controller) is developed and introduced in this paper and applied to the power regulation of a reactor power model. In addition, the test and verification of this application is conducted by two different methods and devices: the virtual verification platform and the physical DCS (digital control system). The result of the verification suggests that the application of SDMC gains a better performance in the maximum dynamic deviation, adjustment time, overshoot, and so on.

\section{Introduction}

In the load tracking operation mode of a PWR (pressurized water reactor) nuclear power plant, the reactor power needs to be dynamically adjusted to track the load demand. The reactor power is highly correlated with the neutron flux in the reactor core. The material of control rod has a large neutron absorption cross section, which means that the control rod has a strong neutron absorption capacity. The proportion of neutrons absorbed by the control rod depends on the depth of the control rod inserted into the core. Therefore, by changing the insertion depth of the control rods, the neutron flux can be adjusted and regulated, and so the reactor power can be controlled.

In a PWR nuclear power plant, the reactor power is controlled by the reactor power control system and the average coolant temperature control system, in which the former one is the main research object in this paper. The function of reactor power control system is to acquire and process related process signals of process system of plant, the state parameter signals of field devices, and the operational command signals from operators. Then, the operation and calculation of logical calculation is conducted in the reactor power control system, from which the output command drives the control rods to regulate the reactor power.

The frame diagram of reactor power control system is shown in Figure 1. The reactor power control system includes two subsystems, the power control system I and power control system II, which control the control rod group $\mathrm{G}$ and control rod group $\mathrm{H}$, respectively. The control modes of control system include automatic control mode and manual control mode. According to the nuclear power and coolant temperature of the primary circuit, the control system determines the control mode. If the prerequisites of automatic mode are met, the reactor power control system is operating in automatic mode; otherwise, it is running in manual mode.

In certain control mode, the two power control systems collect the input signals, conduct the logic calculation of power control, work out the move velocity and direction of control rods, send the command signals to the corresponding rod control and position indication system, and therefore drive the rod to move to regulate the power.

In the case of automatic control mode, the power regulation function is mainly realized by the power control algorithm installed in the power control system. At present, 


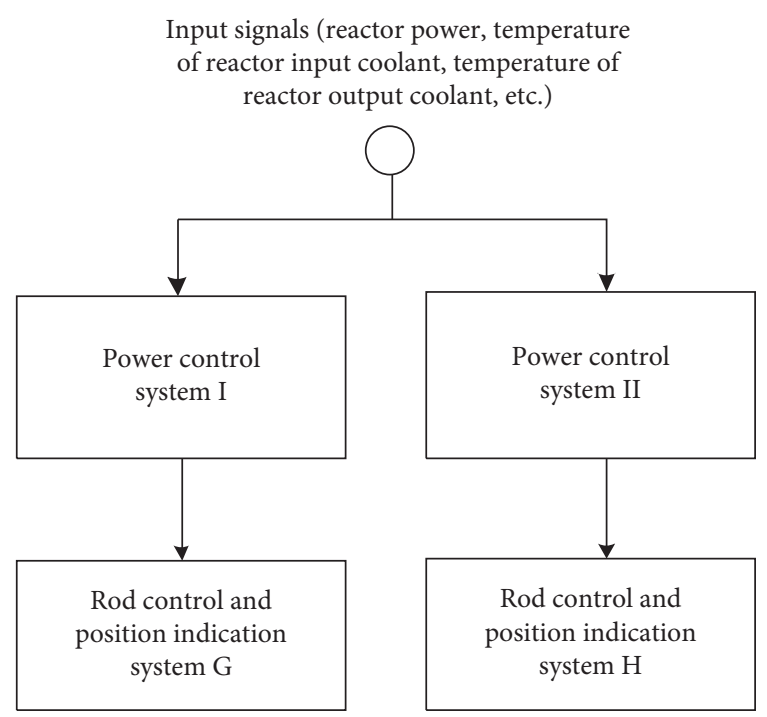

Figure 1: Frame diagram of reactor power control system.

the PID (proportional-integral-derivative) controller [1-3], which has been already widely used in the automation field, is also usually adopted as the power control algorithm. PID controller is flexible and able to meet the basic requirements of nuclear power control system. But there are still some shortcomings like the relatively long response time and large overshoot. Besides, much stringent conditions are required to meet the requirements of automatic mode of reactor power regulation before the automatic mode can be put into operation. These requirements include response time, robustness, and other higher performance factors.

Therefore, recently some researches focused on the control algorithm of reactor power have already been conducted [4-6]. There are many research achievements on the improvement of PID algorithm so far, focusing on the optimization of PID parameters, such as Genetic Algorithm [7-9], Particle Swarm Optimization [10-12], T-S fuzzy identification [10], adaptive PID [13], and other methods, to improve the control performance of PID controller. Another research direction is to use predictive control for PWR reactor power control, mainly to improve the performance of predictive control. And these methods include the combination of predictive control with T-S fuzzy control [14] or self-adaptive method [15], etc. In addition, the predictive control was also applied to the control of coolant average temperature [16]. Besides the PWR, it was also applied to other types of reactors, such as the HTGR (high-temperature gas-cooled reactor) [17].

In this paper, a well-optimized predictive control method with better performance is introduced into the reactor power regulation system, which is proved to be pretty significant for improving the automation level of the reactor control system.

During a control process, the predictive control algorithm is able to predict the future outputs in a certain period of time, and then the predicted outputs are adopted into the control logic to improve its performance $[18,19]$. Therefore, the predictive control algorithm has a better performance in controlling the object featuring time-lag. Three typical and widely used predictive control algorithms include the dynamic matrix control (DMC), generalized predictive control (GPC), and model algorithmic control (MAC). In this paper, a well-optimized predictive control algorithm based on SDMC (stepped dynamic matrix controller) is developed and applied to the power regulation of a core power model [20], and the design of the reactor power control based on the predictive control method is completed. In addition, in order to verify the design of the predictive control algorithm, the simulation verification and physical verification are generally adopted [21-25]. In this paper, the test and verification of the application above is conducted by two different methods and devices: the physical DCS (digital control system) and the virtual verification platform, i.e., the NASPIC platform of nuclear safety class DCS developed by Nuclear Power Institute of China and the virtual DCS of NASPIC. The results show that the stepped dynamic matrix predictive control is pretty applicable to the reactor power control system and gains a better performance in the maximum dynamic deviation, adjustment time, overshoot, and so on.

\section{Reactor Power Controlling Based on Predictive Control Method}

2.1. Design of Stepped Dynamic Matrix Predictive Controller. The SDMC is a predictive control algorithm that adopts step strategy based on the conventional dynamic matrix algorithm to solve the control rate. Define control rate $\Delta u_{t}$ as

$$
\begin{aligned}
\Delta u_{t} & =\delta, \\
\Delta u_{t+i} & =\beta \cdot \Delta u_{t+i-1}=\beta^{i} \cdot \delta, \quad 1 \leq i \leq M-1,
\end{aligned}
$$

where $M$ is the control time domain, $\beta$ is the stepped factor, and $\delta$ is the current control rate.

Then the future control matrix (sequence) can be expressed as

$$
\begin{aligned}
\Delta \mathbf{U}^{T} & =\left(\Delta u_{t}, \Delta u_{t+1}, \ldots, \Delta u_{t+M-1}\right) \\
& =\left(\delta, \beta \delta, \ldots, \beta^{M-1} \delta\right) \\
& =\left(1, \beta, \ldots, \beta^{M-1}\right) \delta
\end{aligned}
$$

As seen from the equation above, the control rate $\Delta \mathbf{U}$ is in a stepped shape with stable and balanced change, which avoids the matrix inversion involved in the solving process of control rate. It greatly simplifies the calculation and improves the stability. And at the same time, the added constraints meet the requirements of actual engineering control, so it is more suitable for engineering application [26].

Set the objective function indicating the deviation of the predicted value from the set value of the target variable and the cost of control rate. As the objective function reaches to its minimum value, the corresponding parameter values are considered as the optimal ones. The objective function $J$ is as follows: 


$$
\begin{aligned}
J & =\sum_{i=0}^{P-1}\left(\widehat{y}_{k+i}-w_{k+i}\right)^{2}+\sum_{i=0}^{M-1} \lambda\left(\Delta u_{k+i}\right)^{2} \\
& =(\widehat{Y}-\mathbf{W})^{T}(\widehat{Y}-\mathbf{W})+\lambda \Delta \mathbf{U}^{T} \Delta \mathbf{U},
\end{aligned}
$$

where $P$ is the predicted time domain; $\widehat{Y}$ and $\mathbf{W}$ are the predicted value matrix and set point value matrix of the target variable, respectively; $k$ indicates the time in a discrete time sequence; and $\lambda$ is the weight of control matrix.

The prediction equation of SDMC involved in the prediction value of the model is as follows:

$$
\widehat{\mathbf{Y}}=\mathbf{S} \Delta \mathbf{U}+\mathbf{Y}_{0},
$$

where $\mathbf{Y}_{0}$ is the initial predicted value matrix and $\mathbf{S}$ is the dynamic matrix committed to achieve the sliding optimization of calculation.

Considering equations (1)-(4), the equivalent dynamic matrix of the stepped predictive control algorithm can be given as follows:

$$
\begin{aligned}
\mathbf{S} \Delta \mathbf{U} & =\left(\begin{array}{cccc}
s_{1} & 0 & \cdots & 0 \\
s_{2} & s_{1} & \cdots & 0 \\
\vdots & & \ddots & \\
s_{M} & s_{M-1} & \cdots & s_{1} \\
\vdots & & & \vdots \\
s_{P} & s_{P-1} & \cdots & s_{P-M+1}
\end{array}\right)\left(\begin{array}{c}
1 \\
\beta \\
\vdots \\
\beta^{M-1}
\end{array}\right) \delta \\
& =\left(\begin{array}{c}
s_{1}+\beta s_{1} \\
\vdots \\
s_{M}+\beta s_{M-1}+\cdots+\beta^{M-1} s_{1} \\
\vdots \\
s_{P}+\beta s_{P-1}+\cdots+\beta^{M-1} s_{P-M+1}
\end{array}\right) \delta \triangleq \mathbf{G} \delta,
\end{aligned}
$$

where $\mathbf{G}$ is a column vector with $P$ in dimension. Therefore, equations (4) and (3) of SDMC algorithm can be given as follows:

$$
\begin{aligned}
\widehat{\mathbf{Y}}= & \mathbf{G} \delta+\mathbf{Y}_{0}, \\
J= & \left(\mathbf{Y}_{0}+\mathbf{G} \delta-\mathbf{W}\right)^{T}\left(\mathbf{Y}_{0}+\mathbf{G} \delta-\mathbf{W}\right)+\lambda \\
& \cdot\left(1+\beta^{2}+\cdots+\beta^{2(M-1)}\right) \delta^{2} .
\end{aligned}
$$

As for the objective function $J$, while $\partial J / \partial \delta=0$, the most optimal value of control rate $\delta$ can be obtained as follows:

$$
\delta=\frac{\mathbf{G}^{T}\left(\mathbf{W}-\mathbf{Y}_{0}\right)}{\mathbf{G}^{T} \mathbf{G}+\lambda\left(1+\beta^{2}+\cdots+\beta^{2(M-1)}\right)} .
$$

As shown in equation (7), control rate $\delta$ is effected by both the weight of control matrix $\lambda$ and the stepped factor $\beta$. $\lambda$ is used to balance the goal of minimizing predictive error and depressing too aggressive control behavior, and a larger $\lambda$ will impose a stronger depression on the fluctuation of control variable. In addition, the value of $\lambda$ is related to the dimension of control variable and controlled variable. In case that $\lambda$ is located in improper range, it will impose almost no effect on $\delta$, even if $\lambda$ zooms in or out in dozens of times of normal value. In the calculation and verification process, this paper deeply analyzed the relationship between the values of related parameters. It can be seen from equation (7), $\lambda$ works only in the denominator of the control equation, which depresses the control value fluctuation. The denominator is dependent on $\lambda$ linearly, in case the rest parameters are fixed. As $\lambda$ decreases, the denominator decreases, and the absolute value of current control rate $\delta$ increases; namely, the control behavior changes dramatically. Conversely, as $\lambda$ increases, the absolute value of current control rate $\delta$ decreases, and the control behavior becomes soft. But it is worth noting that the influence of $\lambda$ depends on the relative size of the two terms in the denominator.

According to the analysis, the range of $\lambda$ and the lower limit value of $\lambda$ were obtained, which further simplified the algorithm to make it more suitable for the application on fast process. The value of stepped factor $\beta$ is around 1 . The response is slowed down when $\beta$ is above 1 and speeded up when $\beta$ is less than 1 .

Finally, the SDMC algorithm was adopted to a graphical control model developed on SCADE software. The control model consists of two parts, the controller based on SDMC algorithm and the controlled object, where the reactor power model was chosen as the controlled object in this research.

2.2. Reactor Power Model. The reactor power model was chosen as the controlled object, referring to [19]. In the reactor power model, the reactor neutron dynamics model, thermal hydraulics model, and reactivity model are taken into consideration. This model also includes measuring instruments and control rods, but not the controllers. The input of the reactor power model is the value of rod velocity, of which the negative value means the descending of control rod, and the positive value means ascending of control rod. In addition, the output of the model is the normalized reactor power value. The model is shown as follows:

$$
\left\{\begin{array}{l}
\dot{\mathbf{x}}=\mathbf{A} \cdot \mathbf{x}+\mathbf{B} \cdot u \\
y=\mathbf{C} \cdot \mathbf{x}
\end{array}\right.
$$

where $\mathbf{x}$ is the variables matrix of state space, indicating the density of neutron, concentration of precursor nucleus, average temperature of fuel, temperature of outlet coolant, and activity change led by the movement of control rod. $\dot{x}$ is the derivative of $\mathbf{x} \cdot y$ is the output of state space, indicating the deviation of the present power from its set point. A, B, and $\mathbf{C}$ are coefficient matrices involved in the setting of model. Besides, $u$ is the input of state space, representing the regulating effect on reactor power introduced by the control rod.

According to the reactor power model, the variables, output, and input of state space are set as follows: 
TABle 1: Data for simulation of PWR plant [26].

\begin{tabular}{|c|c|c|}
\hline Parameters & Values & Explanations \\
\hline$n_{0}$ & $0.5 \mathrm{~m}^{-3}$ & Equilibrium density of neutron \\
\hline$G_{r}$ & 0.0290 & Value of control rod per unit length \\
\hline$P_{0}$ & $2500 \mathrm{MW}$ & Rated power of reactor core \\
\hline$\alpha_{f}$ & $\left(n_{0}-4.24\right) \times 10^{-5 \circ} \mathrm{C}^{-1}$ & Temperature coefficient of reactivity of fuel \\
\hline$\lambda$ & $0.15 \mathrm{~s}^{-1}$ & Decay constant of the delayed neutron \\
\hline$\mu_{c}$ & $\left(160 n_{0} / 9+54\right) \mathrm{MW} \cdot \mathrm{s} \cdot{ }^{\circ} \mathrm{C}^{-1}$ & Heat capacity of coolant \\
\hline$\beta$ & 0.006019 & Total fraction of effective delayed neutron \\
\hline$\Omega$ & $\left(5 n_{0} / 3+4.93\right) \mathrm{MW} \cdot \mathrm{s} \cdot{ }^{\circ} \mathrm{C}^{-1}$ & $\begin{array}{l}\text { Heat transfer coefficient between the fuel and the } \\
\text { coolant }\end{array}$ \\
\hline$f_{f}$ & 0.92 & Fraction of fuel power \\
\hline$M$ & $\left(28 n_{0}+74\right) \mathrm{MW} \cdot \mathrm{s} \cdot{ }^{\circ} \mathrm{C}^{-1}$ & Heat capacity per unit mass of flow \\
\hline$\Lambda$ & $0.00002 \mathrm{~s}$ & Generation time of neutron \\
\hline$\alpha_{c}$ & $\left(-4 n_{0}-17.3\right) \times 10^{-5 \circ} \mathrm{C}^{-1}$ & Temperature coefficient of reactivity of coolant \\
\hline$\mu_{f}$ & $2.63 \mathrm{MW} \cdot \mathrm{s} \cdot{ }^{\circ} \mathrm{C}^{-1}$ & Heat capacity of fuel \\
\hline
\end{tabular}

$$
\begin{aligned}
& \left\{\begin{array}{l}
x=\left(\delta_{n}, \delta_{c}, \delta_{T_{f}}, \delta_{T_{1}}, \delta_{\rho_{r}}\right)^{T}, \\
y=\delta_{n}, \\
u=Z_{r},
\end{array}\right. \\
& \mathbf{A}=\left[\begin{array}{ccccc}
-\frac{\beta}{\Lambda} & \lambda & \frac{\alpha_{f}}{\Lambda} & \frac{\alpha_{c}}{2 \Lambda} & \frac{n_{0}}{\Lambda} \\
\frac{\beta}{\Lambda} & -\lambda & 0 & 0 & 0 \\
\frac{f_{f}}{\mu_{f}} P_{0} & 0 & -\frac{\Omega}{\mu_{f}} & \frac{\Omega}{2 \mu_{f}} & 0 \\
\frac{1-f_{f}}{\mu_{c}} P & 0 & \frac{\Omega}{\mu_{c}} & -\frac{2 M+\Omega}{2 \mu_{c}} & 0 \\
0 & 0 & 0 & 0 & 0
\end{array}\right], \\
& \mathbf{B}^{T}=\left[\begin{array}{lllll}
0 & 0 & 0 & 0 & G_{r}
\end{array}\right], \\
& \mathbf{C}=\left[\begin{array}{lllll}
1 & 0 & 0 & 0 & 0
\end{array}\right],
\end{aligned}
$$

where $\delta_{n}$ is the deviation of neutron density from its equilibrium value, $\mathrm{m}^{-3} . \delta_{c}$ is the change of concentration of precursor nucleus, $m^{-3}$. $\delta_{T f} \delta_{T 1}$, and $\delta \rho_{r}$ represent the activity introduced by average temperature of fuel, temperature of outlet coolant, and movement of control rod, respectively.

The data for simulation of PWR plant are shown in Table 1, according to [26]. Finally, the state space model above was transformed into a transfer function model and then discretized, as shown in the following:

$G\left(z^{-1}\right)=\frac{2.649 z^{-1}-5.812 z^{-2}+4.16 z^{-3}-0.9604 z^{-4}-0.002953 z^{-5}}{1-3.055 z^{-1}+3.439 z^{-2}-1.689 z^{-4}+1.096 e^{-17} z^{-5}}$.

Actual power control system object has a certain nonlinear characteristic. But the predictive control has the function of feedback correction; namely, the prediction model (liner model) is able to do the real time correction on control output vector based on both the history output and actual output. Therefore, the predictive control has low requirement for the accuracy of model and has a better robust characteristic, which can overcome the nonlinear characteristic to some degree.

2.3. Stability Analysis of Control System. The stability analysis is applied by converting the stepped dynamic matrix predictive control into an internal model control structure. The specific analysis process is as follows.

2.3.1. Internal Model Controller and Stability Lemma. The principle structure of internal model control is shown in Figure 2. $G_{p}\left(z^{-1}\right)$ is the actual object transfer function, $G_{m}\left(z^{-1}\right)$ is the identification object transfer function, $G_{c}\left(z^{-1}\right)$ is the controller, $F\left(z^{-1}\right)$ is the filter which meets the condition $\lim F\left(z^{-1}\right)=1, r(t)$ is the reference input, and $d(t)$ is the disturbance.

From the lemmas of internal model control, the stability theorem of internal model control structure of SDMC can be obtained.

Lemma 1. If the SDMC system is stable, there is no static deviation between the output $y(t)$ and the set value $r$.

Lemma 2. For the control system in Figure 2, when the actual object is in exact agreement with the identification object, a sufficient and necessary condition for the stability of the closed-loop system is that both the controller and the object are stable.

Lemma 3. If the internal model control system shown in Figure 2 is closed-loop stable, the sufficient and necessary condition for no static deviation in the steady state of the system is $G_{c}(1)=G_{m}^{-1}(1)$.

Lemmas 1 and 2 can be easily proved, and the proof of Lemma 3 is as follows.

The closed-loop transfer function is 


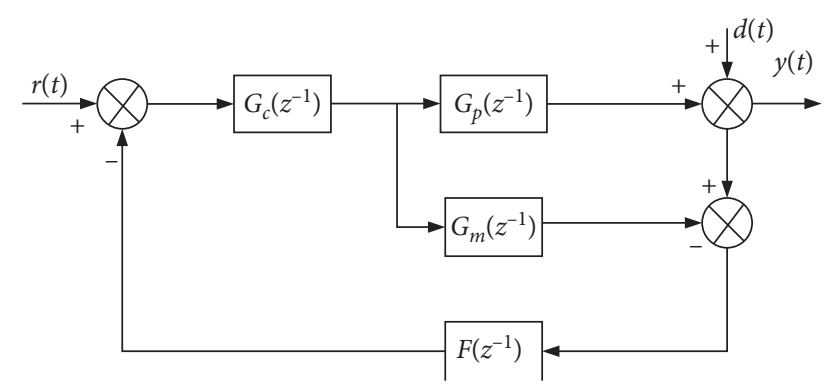

Figure 2: The principle structure of internal model control.

$$
\frac{y\left(z^{-1}\right)}{r\left(z^{-1}\right)}=\frac{G_{c}\left(z^{-1}\right) G_{p}\left(z^{-1}\right)}{1+G_{c}\left(z^{-1}\right) F\left(z^{-1}\right)\left[G_{p}\left(z^{-1}\right)-G_{m}\left(z^{-1}\right)\right]} .
$$

If $G_{c}(1)=G_{m}^{-1}(1)$, the steady-state output (with an unit step reference input) is

$$
\begin{aligned}
y_{r}(\infty)= & \lim _{z \longrightarrow 1} \frac{G_{c}\left(z^{-1}\right) G_{p}\left(z^{-1}\right)}{1+G_{c}\left(z^{-1}\right) F\left(z^{-1}\right)\left[G_{p}\left(z^{-1}\right)-G_{m}\left(z^{-1}\right)\right]} \\
& =\frac{G_{c}(1) G_{p}(1)}{1-G_{c}(1) G_{m}(1)+G_{c}(1) G_{p}(1)}=1 .
\end{aligned}
$$

Therefore, there is no static deviation between the output $y$ and the reference input, which can also prove that there is also no static deviation between the output $y$ and the disturbance $d$; i.e.,

$$
\lim _{t \longrightarrow \infty} y_{d}(t)=0
$$

On the other hand, if there is no static difference in the steady state of the system, then

$$
y_{r}(\infty)=\frac{G_{c}(1) G_{p}(1)}{1-G_{c}(1) G_{m}(1)+G_{c}(1) G_{p}(1)}=1 .
$$

Therefore, it can be proved that $G_{c}(1) G_{m}(1)=1$, and Lemma 3 can be obtained.

2.3.2. Proof of Stability of SDMC. The internal model control structure of SDMC is shown in Figure 3, which corresponds to the standard internal model control structure.

The filter and controller of SDMC are $F\left(z^{-1}\right)=1$, $H_{c}\left(z^{-1}\right)=G_{c}\left(z^{-1}\right)$, where $H_{c}\left(z^{-1}\right)$ is a polynomial of $N$ orders called SDMC polynomial. Define

$$
\begin{aligned}
& H_{c}\left(z^{-1}\right)=h_{c 0}+h_{c 1} z^{-1}+\cdots+h_{c N} z^{-N}=\sum_{i=1}^{N} h_{i} z^{-i}, \\
& H^{*}\left(z^{-1}\right)=\sum_{i=1}^{\infty} h_{i}^{*} z^{-i},
\end{aligned}
$$

where $H^{*}\left(z^{-1}\right)$ is the actual transfer function, $H^{*}\left(z^{-1}\right)$ is the step response measured from system, and $N$ is the truncated step size of the model (modeling time domain).

The following theorem will adopt SDMC polynomial to assess the stability of the controller and the system.

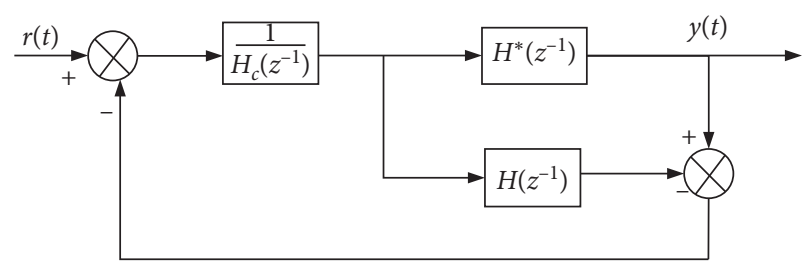

FIgURE 3: The internal model control structure of SDMC.

Proposition 1. In the SDMC structure shown in Figure 3, SDMC polynomial $H_{c}\left(z^{-1}\right)$ meets $H_{c}(1)=H(1)$.

It is proved that there is no static deviation in steady state of SDMC system according to Lemma 1, and according to Lemma 3, it can be derived that

$$
H_{c}(1)=H(1)=\sum_{i=1}^{N} h_{i}=a_{N},
$$

where $a_{0}, a_{1}, \ldots, a_{N}$ are the step response coefficients of the object. Therefore, the theorem can be obtained.

Theorem 1. When the model error is small, for the stable control system as shown in Figure 3, the necessary and sufficient condition is that the roots of SDMC polynomial $H_{c}\left(z^{-1}\right)$ are in a unit circle.

The proof is as follows. According to Lemma 2, when $H^{*}\left(z^{-1}\right)=H\left(z^{-1}\right)$, the sufficient and necessary condition for the stability of the closed-loop system is that both the controller and the object are stable. Since SDMC is only applied to the stable system, the sufficient and necessary condition for closed-loop stability is determined by the position of the poles of $1 / H_{c}\left(z^{-1}\right)$. Therefore, the theorem is proved.

Theorem 2. If the steady-state gain is greater than 0 , the necessary condition for the stability of the SDMC polynomial is $H_{c}(\infty)=h_{c 0}>0$.

The proof is as follows. According to Proposition 1, $H_{c}(1)=H(1)$, and it is worth noting that $H(1)$ represents the steady-state value of the step response curve of the model. Therefore,

$$
H(1)=a_{N}>0
$$

If $H(\infty)<0$, because $H_{c}\left(z^{-1}\right)$ is analytic in the complex plane except for the origin point, then there is at least one root of $H_{c}\left(z^{-1}\right)=0$ that falls at the range $(0, \infty)$, and the closed-loop system is unstable. If $H_{c}(\infty)=h_{c 0}=0$, the governing equation is

$$
u(t)=\frac{r(t)}{h_{c 0}}-\frac{y(t)-y_{m}(t)}{h_{c 0}}-\frac{1}{h_{c 0}} \sum_{i=1}^{N} h_{c i} u(t-i),
$$

where the denominator is zero and is meaningless.

In summary, the necessary condition for the stability of $H_{c}\left(z^{-1}\right)$ is $h_{c 0}>0$, and Theorem 2 is proved.

For equation (18), the input and output are always controlled to change in the same direction. Therefore, in 
equation (17), $a^{N}$ is actually not equal to 0 , which meets the requirement of reactor power control system.

Theorem 3. The sufficient condition of the root of polynomial $a_{0}+a_{1} z^{-1}+\cdots+a_{N} z^{-N}\left(a_{0}<0\right)$ that falls in the unit circle is $\sum_{i=1}^{N}\left|a_{i}\right|<a_{0}$.

The proof is as follows. For $|z| \geq 1$, there is

$$
\left|a_{0}+a_{1} z^{-1}+\cdots+a_{N} z^{-N}\right| \geq a_{0}-\sum_{i=1}^{N}\left|a_{i}\right|\left|z^{-i}\right| \geq a_{0}-\sum_{i=1}^{N}\left|a_{i}\right|>0
$$

so there is no root that falls outside the unit circle, and Theorem 3 is proved.

\subsection{Reactor Power Control Based on Predictive Controller.} The optimized predictive control algorithm, SDMC, is introduced into the power regulation control system, with the reactor power model being the controlled object, to realize the function of reactor power control based on predictive control.

The composition of the reactor power control platform is shown in Figure 4. The reactor power control platform receives data from nuclear measurement system, the reactor protection system, rod control and position system, and the main control room. Then, SDMC algorithm which is written into the distributed processing unit (DPU) of the reactor power regulation system responds to the input signals and produces output signals that represent the velocity and direction of the rods. Finally, the output signals are sent to the $G$ and $\mathrm{H}$ rod groups and other parts that require the signals (e.g., the nonclassified DCS section and the main control room).

SDMC was composed in NASLAD software, which is a part of NASPIC platform configuration software. The NASLAD software is shown in Figure 5(a). The framework of SDMC and its inputs and outputs are shown in Figure 5(b), where the SDMC function block is encapsulated into the algorithm block named SDMC.

The inputs of SDMC algorithm block include the normalized set point value (SP) of reactor power received by analogy input (AI) module, and the feedback value of power (PV) from the controlled object (reactor power model) received by AI module. The output of SDMC algorithm block is named $U$, which is transferred to the controlled object. In addition, the SDMC algorithm block also receives input of parameter variables, including $u \_d n, d u \_d n$, u_initvalue, u_up, and du_up, which represent the lower limit of control quantity, lower limit of control rate, initial value of control quantity, upper limit of control quantity, and upper limit of control rate, respectively. The algorithm block named HTI will translate the format of parameters in the control process.

In addition, the control principle block diagram of SDMC algorithm block is shown in Figure 5(c). The internal logic diagram of SDMC controller mainly consists of three parts: prediction model, rolling optimization, and feedback correction. In Figure 5(c), $\mathbf{w}_{\mathbf{p}}$ is the vector of inputs for

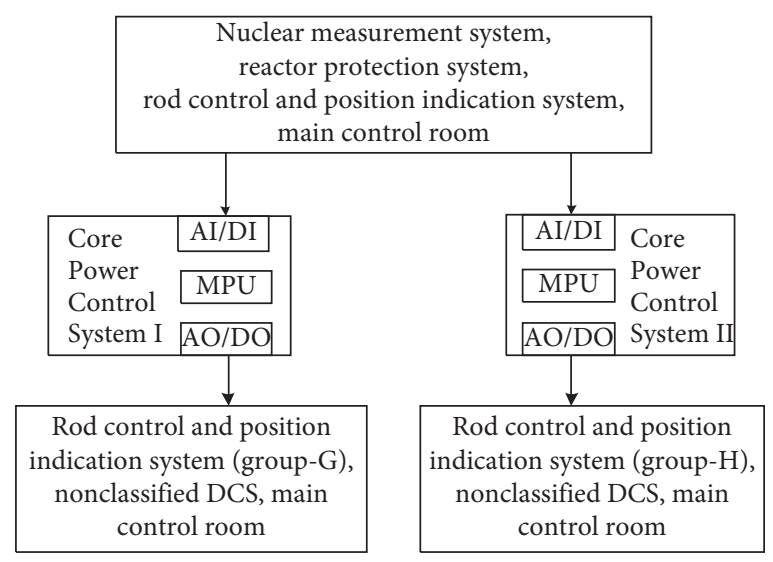

Figure 4: Composition of the reactor power control platform.

SDMC, $y$ is the real output, $\boldsymbol{Y}_{\mathrm{P} 0}$ is the prediction value in prediction time domain, $\hat{y}$ is the revised prediction vector, and $e$ is the deviation between $y$ and $\hat{y}$.

After the composition in the software NASLAND, the power regulation algorithm software will be installed by the maintenance station into the physical platform of DCS or virtual DCS. And the DCS installed with the power regulation algorithm software will realize the control and regulation function. According to the design of the NASPIC platform, the values and states of related signals (variables) of it can be monitored as the DCS is in maintenance mode.

\section{Verification of Control Algorithm}

The verification of SDMC algorithm for the reactor power control system was conducted by two different methods and devices: the virtual verification platform and the physical DCS.

3.1. Virtual Platform for Verification. The virtual testing and verifying platform consists of the virtual DCS of NASPIC and the maintenance station (NASPES) of NASPIC.

The tasks performed by NASPES mainly include the software configuration, the software compilation, the software installation, the operation monitoring, and other functions. Software configuration refers to designing the engineering file which carries the logic algorithm at the maintenance station. Software compilation refers to compiling the engineering file into the executable file for DCS. Software installation refers to transmitting and installing the executable file to DCS. Operation monitoring refers to the monitoring of the logical operation status of DCS by maintenance station.

Besides the maintenance station, another part of virtual testing and verifying platform is the virtual DCS. The virtual DCS is the virtualization simulation of physical platform of DCS for NASPIC, developed by Nuclear Power Institute of China. The virtual DCS runs in Microsoft Windows system. The core of virtual DCS is a software named virtual distributed processing unit (VDPU), which is the virtualization simulation of physical DPU in a physical DCS. VDPU is to simulate the performance and function of DPU and finally 


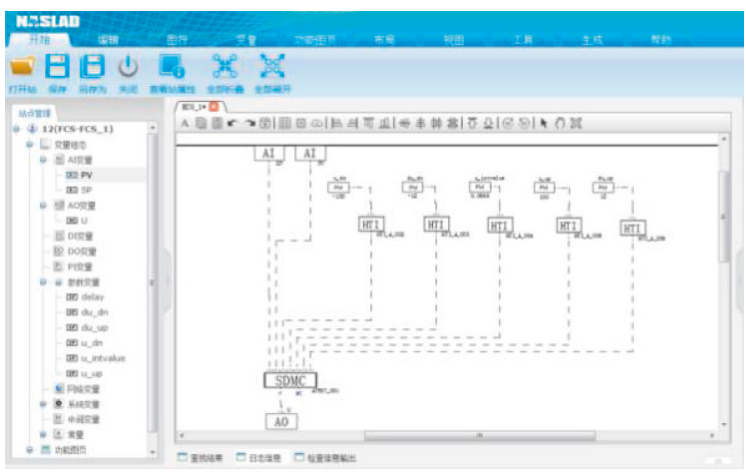

(a)

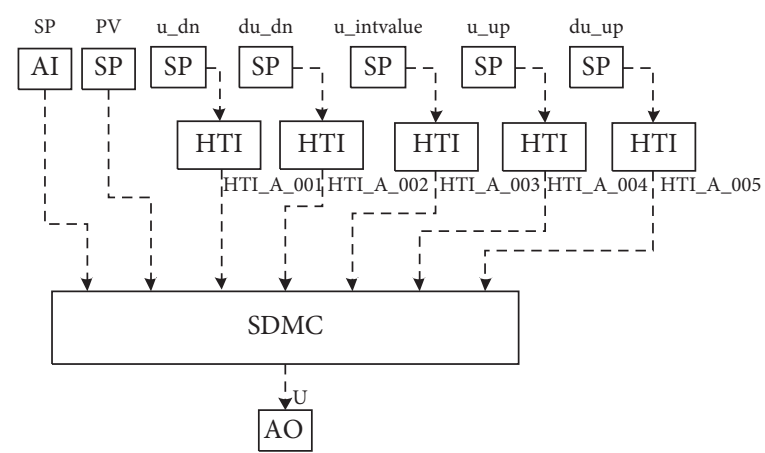

(b)

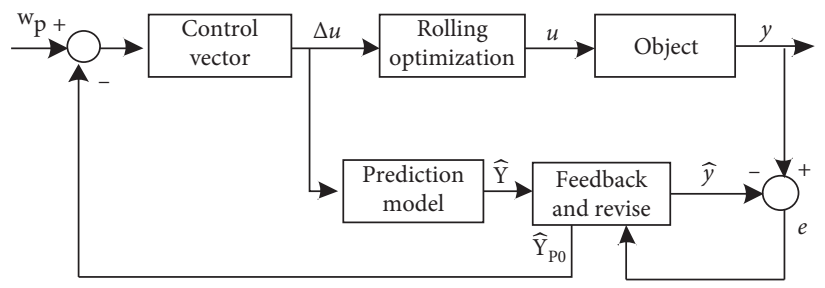

(c)

FIgURE 5: SDMC Logic in NASLAD. (a) NASLAD software. (b) Framework of SDMC. (c) Control principle block diagram of SDMC.

execute the simulation tasks of the physical DCS. The main components of virtual DCS are shown in Figure 6, among which the user management interface (UMI) is developed as the interactive interface for operator, the VDPU is responsible for the algorithm operation, the dispatch management service (DMS) is for data management and scheduling, and the virtual processing station (VPS) is for the network communication between DMS and VDPU. Virtual DCS and physical DCS are installed with the same algorithm software, and their data source is exactly the same, and they just only differ in the format of the executable files. Therefore, the exact consistency of functions of them can be ensured.

3.2. Physical DCS for Verification. The physical testing and verifying platform for reactor power control algorithm verification consists of nuclear safety class DCS (NASPIC), signal generator, oscilloscope, and maintenance station (NASPES), as shown in Figures 7 and 8. NASPIC includes protection instrumentation preprocessing station and main processing station (consisting of DPU, AI, and $\mathrm{AO}$ ). $\mathrm{AO}$ is the analogy output module. The executable file of logic runs in DPU of the main processing station, where the SDMC algorithm is installed.

Signal generator generates the signal of set point value (SP), which is transmitted to the protection instrumentation preprocessing station, and so is the feedback signal (PV) of process model. After the preprocessing, the signals are sent to the DPU. DPU accepts all of the input signals and executes the algorithm logic. And then, the output signal $(U)$ of controller is transmitted to the process model. And the output feedback of process model is also monitored by oscilloscope.

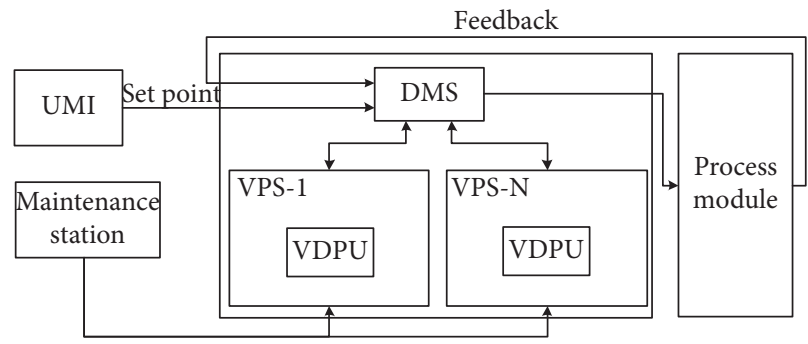

FIgURE 6: Diagram of virtual DCS.

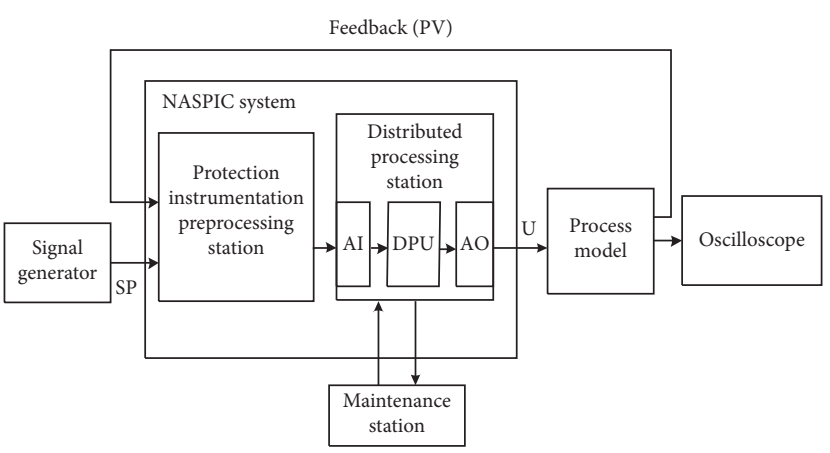

Figure 7: Diagram of physical test platform.

3.3. Result Analysis of Verification. In equation (7), the dynamic matrix $\mathbf{G}$ is composed of the step response coefficients of the object, and in the case where the object model of the system is determined, $\mathbf{G}$ is fixed. In addition, the stepped factor $\beta$ makes the future control rate increase proportionally. $\beta$ corresponds to the distribution of control tasks. A smaller $\beta$ means more emphasis on the function of the former control variables in 


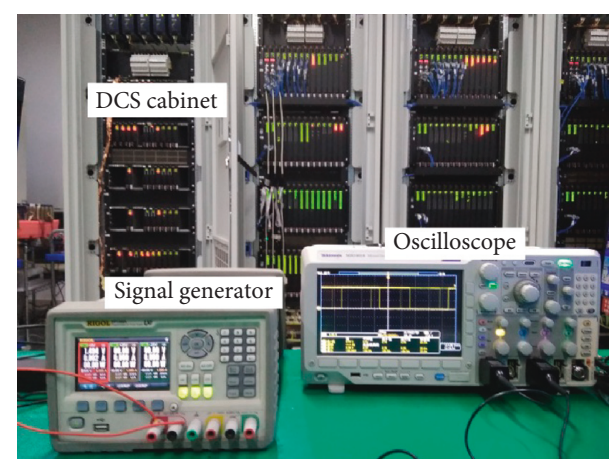

Figure 8: Photo of physical test platform.

sequence, which leads to a faster system response. On the contrary, a larger $\beta$ means more emphasis on the later control variables, delaying control behavior and slowing system response.

Since the predicted output of $P$ (predicted time domain) is only affected by control variables of $M$ (predicted time domain) at most, it can be concluded that $M \leq P$. A larger $P$ means that a longer-term consideration is taken into the prediction, and the predicted output of the future longer period is expected to be close to the expected output. Therefore, the system stability can be better, but the dynamic response process would be slower. The size of the control step $M$ reflects the expectation that $M$ steps of control are able to complete the control task. Therefore, a larger $M$ indicates that the prediction and optimization task is shared by more multiple control variables with strong flexibility, but the stability and robustness of the system become worse.

As for the parameter tuning of $\lambda$, the relative size of two numbers can be expressed by the following ratio:

$$
\begin{aligned}
& n=\frac{\mathbf{G}^{T} \mathbf{G}}{\mathbf{B}^{T} \mathbf{B}}, \\
& m=\frac{\mathbf{G}^{T} \mathbf{G}}{\lambda \cdot \mathbf{B}^{T} \mathbf{B}}=\frac{n}{\lambda},
\end{aligned}
$$

where $\quad \mathbf{B}=\left[1, \quad \beta, \quad \beta^{2}, \quad \ldots, \quad \beta^{M-1}\right], \quad$ and $\quad \mathbf{B}^{T} \mathbf{B}=$ $1+\beta^{2}+\cdots+\beta^{2(M-1)}$. So equation (7) can be rewritten as

$$
\begin{aligned}
\delta & =\frac{\mathbf{G}^{T}\left(\mathbf{W}-\mathbf{Y}_{0}\right)}{\mathbf{G}^{T} \mathbf{G}+\lambda \mathbf{B}^{T} \mathbf{B}} \\
& =\frac{\mathbf{G}^{T}\left(\mathbf{W}-\mathbf{Y}_{0}\right)}{(1+1 / m) \mathbf{G}^{T} \mathbf{G}} \\
& =\frac{\mathbf{G}^{T}\left(\mathbf{W}-\mathbf{Y}_{0}\right)}{(1+m) \lambda \cdot \mathbf{B}^{T} \mathbf{B}} .
\end{aligned}
$$

According to equation (21), while $m>>1,1 / m \approx 0$, item $\lambda \mathbf{B}^{T} \mathbf{B}$ can be negligible. While $m<<1$, the item $\mathbf{G}^{T} \mathbf{G}$ can be ignored. When $m$ is near 1 , the two terms are equal, and ignoring either of them will cause a large error. $\sigma$ is defined to indicate the influence of the neglected term. If $m$ is greater than or equal to $m_{0}$, the relative error is

$$
\begin{aligned}
\sigma & =\left|\frac{\left(\mathbf{G}^{T}\left(\mathbf{W}-\mathbf{Y}_{0}\right) / \mathbf{G}^{T} \mathbf{G}\right)-\mathbf{G}^{T}\left(\mathbf{W}-\mathbf{Y}_{0}\right) /(1+(1 / m)) \mathbf{G}^{T} \mathbf{G}}{\mathbf{G}^{T}\left(\mathbf{W}-\mathbf{Y}_{0}\right) /(1+(1 / m)) \mathbf{G}^{T} \mathbf{G}}\right| \\
& =\left|\frac{1-(1 / 1+(1 / m))}{1 / 1+(1 / m)}\right|=\frac{1}{m} \leq \frac{1}{m_{0}}
\end{aligned}
$$

When $m \leq 1 / m_{0}$ and the term $\mathbf{G}^{T} \mathbf{G}$ is ignored, the relative error is

$$
\begin{aligned}
\sigma & =\left|\frac{\left(\mathbf{G}^{T}\left(\mathbf{W}-\mathbf{Y}_{0}\right) / \lambda \mathbf{B}^{T} \mathbf{B}\right)-\left(\mathbf{G}^{T}\left(\mathbf{W}-\mathbf{Y}_{0}\right) /(1+m) \lambda \cdot \mathbf{B}^{T} \mathbf{B}\right)}{\mathbf{G}^{T}\left(\mathbf{W}-\mathbf{Y}_{0}\right) /(1+m) \lambda \cdot \mathbf{B}^{T} \mathbf{B}}\right| \\
& =\left|\frac{1-1 / 1+m}{1 / 1+m}\right|=m \leq \frac{1}{m_{0}} .
\end{aligned}
$$

In the analysis, the value of $m_{0}$ is usually determined according to the characteristics of the controlled process and the relative error. Therefore, only if $m<m_{0}$, or $\lambda>n / m_{0}$, is $\lambda$ able to effectively depress the fluctuation of control variable. If $\lambda \leq n / m_{0}$, the term $\lambda \mathbf{B}^{T} \mathbf{B}$ is negligible, and $\lambda$ does not work. On the contrary, if $\lambda \geq n / m_{0}$, the term $\mathbf{G}^{T} \mathbf{G}$ is negligible, and the influence of $\lambda$ is significant.

With the reactor power model being adopted as the process model, the SDMC applied on the reactor power control was verified based on 4 typical operating conditions, including the start-up of reactor, lifting and reducing of load, fluctuation of load in wide range, and load shedding. Based on the parameter tuning and verification, the simulating time domain $N$ of the simulation model was set at 200 , the time domain of prediction $P$ at 55, the control time domain $M$ at 4 , and the weight of control matrix $\lambda$ at 0.9 , and the stepped factor $\beta$ at 1.2. It is worth noting that the relative value of reactor power was adopted instead of the absolute reactor power, considering the simplification of the verification and understanding.

The verification of 4 typical operating conditions was conducted based on 3 verification methods, results of which can be seen from Figure 9, where legend SP indicates the set point value of reactor power, and legend SCADE indicates the verification based on SCADE software, legend VDCS on virtual DCS, and legend PDCS on physical DCS. It can be found that the responses of reactor power tracing the set point value SP are in an approximately similar trend. In addition, the performances of different methods are further presented quantitatively in Table 2. Accordingly, it suggests that the responses of reactor power on the 3 different methods have a satisfied agreement, with just a certain deviation as a result of the difference in verification platform and corresponding measure method. It reflected the reliability of SDMC and the verification methods. Thus, it is reasonable that the verification methods are used in the comparison of different control methods. Therefore, the adjustment time and overshoot can be analyzed to compare the different control methods in the instruction below.

In order to further verify the advantage of SDMC algorithm, it was compared with PID and Fuzzy-PID (FPID) by simulation, with the same reactor power model and same 

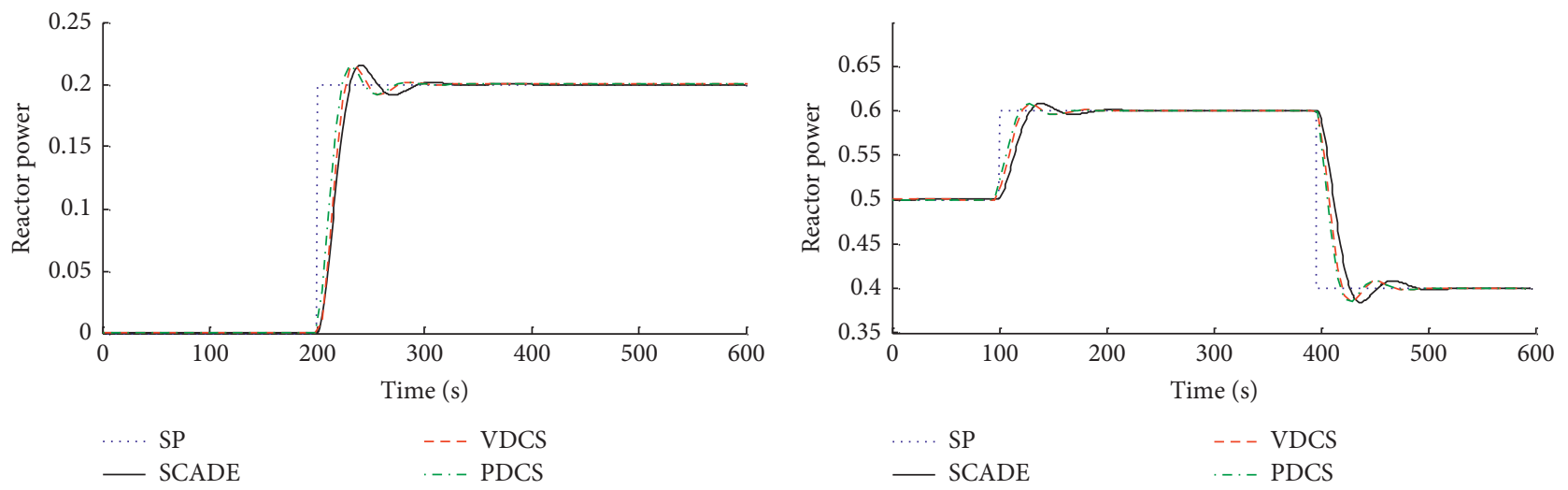

(a)

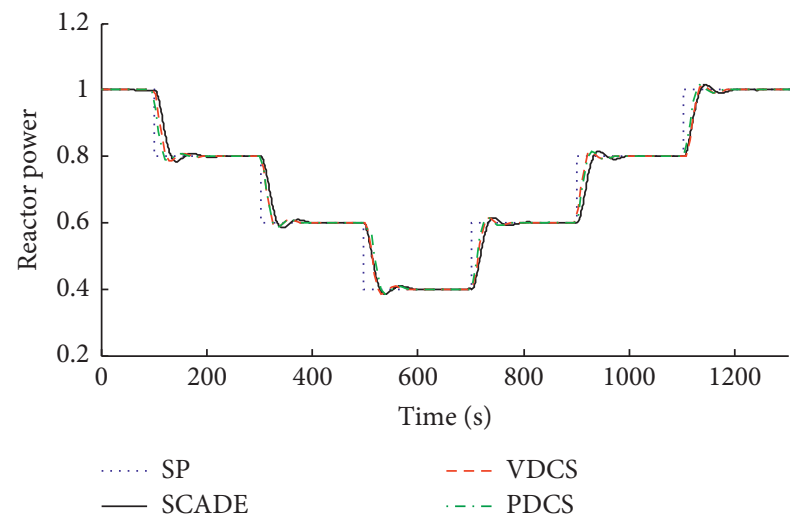

(c)

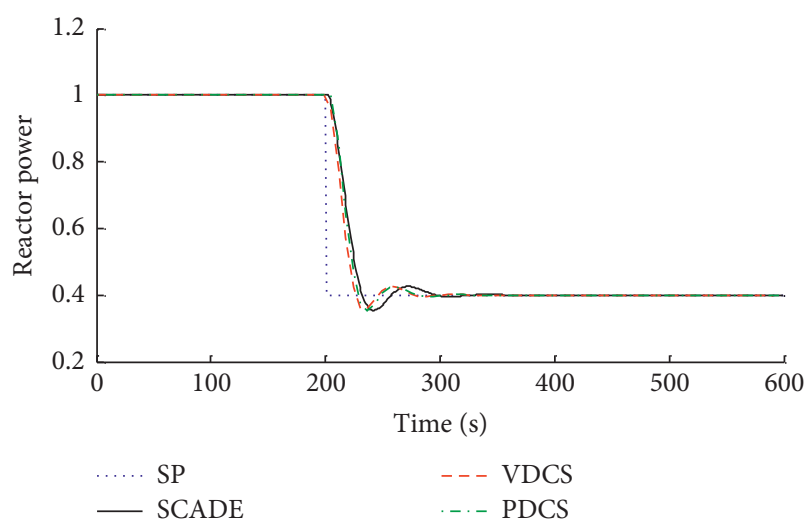

(d)

Figure 9: Verification results on different conditions. (a) Start-up of reactor. (b) Lifting and reducing of load. (c) Fluctuation of load in wide range. (d) Load shedding.

TABLE 2: Comparison of different verification methods.

\begin{tabular}{lcccccc}
\hline \multirow{2}{*}{ Operating conditions } & \multicolumn{3}{c}{ Adjustment time (s) } & \multicolumn{3}{c}{ Overshoot (\%) } \\
& SCADE & VDCS & PDCS & SCADE & VDCS & 7.822 \\
Start-up & 53 & 37 & 36 & 7.832 & 7.821 \\
Lifting and reducing load & 46 & 33 & 34 & 7.801 & 7.641 \\
Fluctuation of load & 42 & 33 & 32 & 0.772 & 0.763 \\
Load shedding & 52 & 39 & 38 & 0.765 & 0.775 \\
\hline
\end{tabular}

operating condition. The change of set point (SP) value with time and corresponding responses of reactor power tracing SP are shown in Figure 10. It shows that the responses of reactor power tracing the set point value SP share a similar change trend. In addition, the performances of different algorithms are further presented quantitatively in Table 3, where ITAE is the integral of time-weighted absolute value of the error. It can be concluded that, in respect of maximum dynamic deviation, overshoot, adjustment time, and ITAE, all the values of SDMC algorithm are lower than those of PID and FPID, indicating that the performance of SDMC is much better. In particular, compared with PID, the advantage of SDMC is absolutely not negligible. Therefore, the SDMC further proves an effective and advantaged algorithm on the control of reactor power.

It can be seen from Table 3 that the steady-state error of each control method is acceptable, indicating that the system

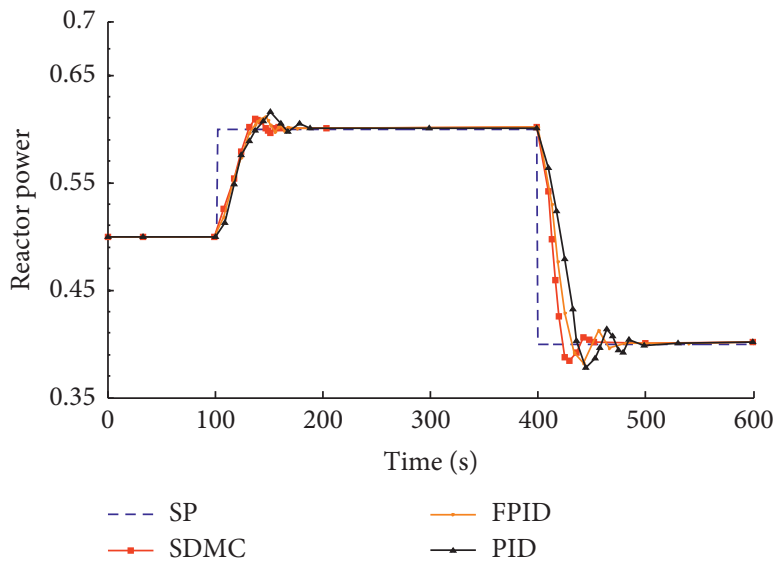

FIGURE 10: Comparison of different control algorithms. 
TABLE 3: Comparison of different control algorithms.

\begin{tabular}{lccccc}
\hline Control method & Maximum dynamic deviation & Overshoot (\%) & Adjustment time (s) & ITAE/1 & Steady-state error (\%) \\
\hline SDMC & 0.013 & 7.898 & 33 & 0.616 & 0.45 \\
PID & 0.021 & 13.00 & 55 & 0.752 & 0.66 \\
FPID & 0.015 & 7.925 & 47 & 0.624 & 0.53 \\
\hline
\end{tabular}

has good steady-state performance under the action of each control method. In addition, SDMC algorithm has better steady-state performance with a relatively smaller steadystate error.

\section{Conclusion}

(1) A well-optimized predictive control algorithm, SDMC, was developed and applied to the power regulation of a reactor power model.

(2) The test and verification of the application of SDMC algorithm on reactor power was conducted by both the virtual verification platform and the physical DCS. The verification results suggested that the dynamic response of reactor power on different verification methods was in good agreement, which reflected the reliability of both the SDMC algorithm and the verification methods.

(3) It can be concluded from the comparison of SDMC, PID, and FPID algorithms by simulation that SDMC algorithm presented a better performance in respect of maximum dynamic deviation, adjustment time, overshoot, and ITAE, reflecting the advantage of SDMC.

\section{Data Availability}

The data used to support the findings of this study are included within the article, and they are mainly presented as figures and tables. Furthermore, data can be accessed from the corresponding author by e-mail.

\section{Conflicts of Interest}

The authors declare that they have no conflicts of interest.

\section{References}

[1] B. Li and X.-X. He, "A reactor power control system," Industry Control and Applications, vol. 27, no. 10, pp. 15-18, 2008.

[2] W.-J. Liu and J.-H. Ye, "Study on power control of nuclear reactors," Journal of Chinese Society of Power Engineering, vol. 36, no. 5, pp. 378-382, 2016.

[3] Q.-Q. Wang, C.-C. Yin, X.-J. Sun et al., "PID design and simulation of TMSR nuclear power control system," Nuclear Techniques, vol. 38, no. 2, 2015.

[4] H.-X. Wu, F.-Y. Zhao, and Z.-M. Zhang, "Application of a new type fuzzy controller in power control system for nuclear reactors," Nuclear Power Engineering, vol. 29, no. 5, 2008.

[5] W.-W. Han, X.-C. Luan, A.-M Yang, and T.-S. Fuzzy, "Integral control system of nuclear reactor power based on fuzzy state observe," Atomic Energy Science and Techno Logy, vol. 47, no. 9, pp. 1572-1578, 2013.
[6] L. Liu, X.-C. Luan, S. Rao, G. Y. Jin, and T. Tu, "Application of robust fuzzy control," Atomic Energy Science and Technology, vol. 47, no. 4, pp. 624-629, 2013.

[7] M. H. S. M. Muzzammil and E. A. Mohamed Ali, "Safety improvement of nuclear power reactor using soft computing techniques," in Proceedings of the International Conference on Energy Efficient Technologies for Sustainability, IEEE, Nagercoil, India, April 2013.

[8] S. M. H. Mousakazemi, N. Ayoobian, and G. R. Ansarifar, "Control of the reactor core power in PWR using optimized PID controller with the real-coded GA," Annals of Nuclear Energy, vol. 118, pp. 107-121, 2018.

[9] S. M. H. Mousakazemi, "Control of a PWR nuclear reactor core power using scheduled PID controller with GA, based on two-point kinetics model and adaptive disturbance rejection system," Annals of Nuclear Energy, vol. 129, 2019.

[10] M.-W. Zhao, Z. Chen, L.-T. Liao et al., "Research on integrated reactor power control system based on intelligent prediction," Nuclear Power Engineering, vol. 40, no. 4, pp. 166-171, 2019.

[11] L.-T. Liao, Z. Chen, and M.-W. Zhao, "The research of generalized intelligent controller design for reactor power control system," Automation \& Instrumentation, no. 4, pp. 229-232, 2019.

[12] S. M. H. Mousakazemi, N. Ayoobian, and G. R. Ansarifar, "Control of the pressurized water nuclear reactors power using optimized proportional-integral-derivative controller with particle swarm optimization algorithm," Nuclear Engineering and Technology, vol. 50, no. 6, 2018.

[13] Z. Dong, "Adaptive proportional-differential power-level control for pressurized water reactors," IEEE Transactions on Nuclear Science, vol. 61, no. 2, pp. 912-920, 2014.

[14] T.-H. Wu, X.-C. Luan, J.-L. Wang et al., "Fuzzy generalized predictive control of the nuclear reactor power," Nuclear Science and Engineering, vol. 36, no. 3, pp. 299-305, 2016.

[15] J. Su, G.-Q. Xia, and W. Zhang, "The study on applying the algorithm of global predictive control with self-adaptive in the power control of the nuclear reactor," Chinese Journal of Nuclear Science and Engineering, vol. 26, no. 4, pp. 289-292, 2006.

[16] H. Qian, Z.-L. Fazng, W.-X. Jin et al., "Protective control and simulation research on nuclear power plant coolant average temperature," Nuclear Power Engineering, vol. 37, no. 2, pp. 91-96, 2016.

[17] H. Shen, J. Zhao, and Z.-H. Xu, "Nonlinear model predictive control of HTR-PM load changing process," Computers and Applied Chemistry, vol. 29, no. 1, pp. 49-55, 2012.

[18] D. Jiang and X.-J. Liu, "Model predictive control of nuclear reactor power based on lexicographic optimization," Control Engineering of China, 2018.

[19] G.-X. Wang, J. Wu, B.-F. Zeng, Z.-B. Xu, W.-Q. Wu, and X.-Q. Ma, "Model predictive control method for core power control in pressurized water reactor," Atomic Energy Science and Technology, vol. 51, no. 3, pp. 480-484, 2017.

[20] T. Fang, D.-G. Lu, and T.-Y. Duan, "Fast breeder reactor power manipulation strategy based on advanced genetic 
programming approach," Journal of Tianjin University, vol. 45, no. 12, pp. 1105-1109, 2012.

[21] H. Lin, M. Lin, D. Hou, and Y.-H. Yang, "Modeling of reactor power control system and closed loop verification," Nuclear Power Engineering, vol. 30, no. 4, pp. 96-99, 2009.

[22] J.-L. Sun, G.-Q. Xia, and F. Sun, "Application of $H_{\infty}$ control method to nuclear reactor power control system," Applied Science and Technology, vol. 32, no. 8, 2005.

[23] J.-T. Feng, X.-J. Huang, and L.-J. Zhang, "Modeling and simulating of power regulating system of a research reactor," Nuclear Power Engineering, no. 3, pp. 92-96, 2006.

[24] L.-T. Liao and Z. Chen, "Study on nuclear reactor power control based on T-S neuro-fuzzy network," Nuclear Power Engineering, vol. 34, no. 3, pp. 109-112, 2013.

[25] J.-T. Feng, X.-J. Huang, and L.-J. Zhang, "Study on characteristic of reactor power regulating system," Atomic Energy Science and Technology, vol. 40, no. 3, pp. 306-310, 2006.

[26] G.-J. Luo, Reseaqrch on the Tuning of Parameters of Stepped Predictive Controller, University of Science and Technology of China, Hefei, China, 2016. 

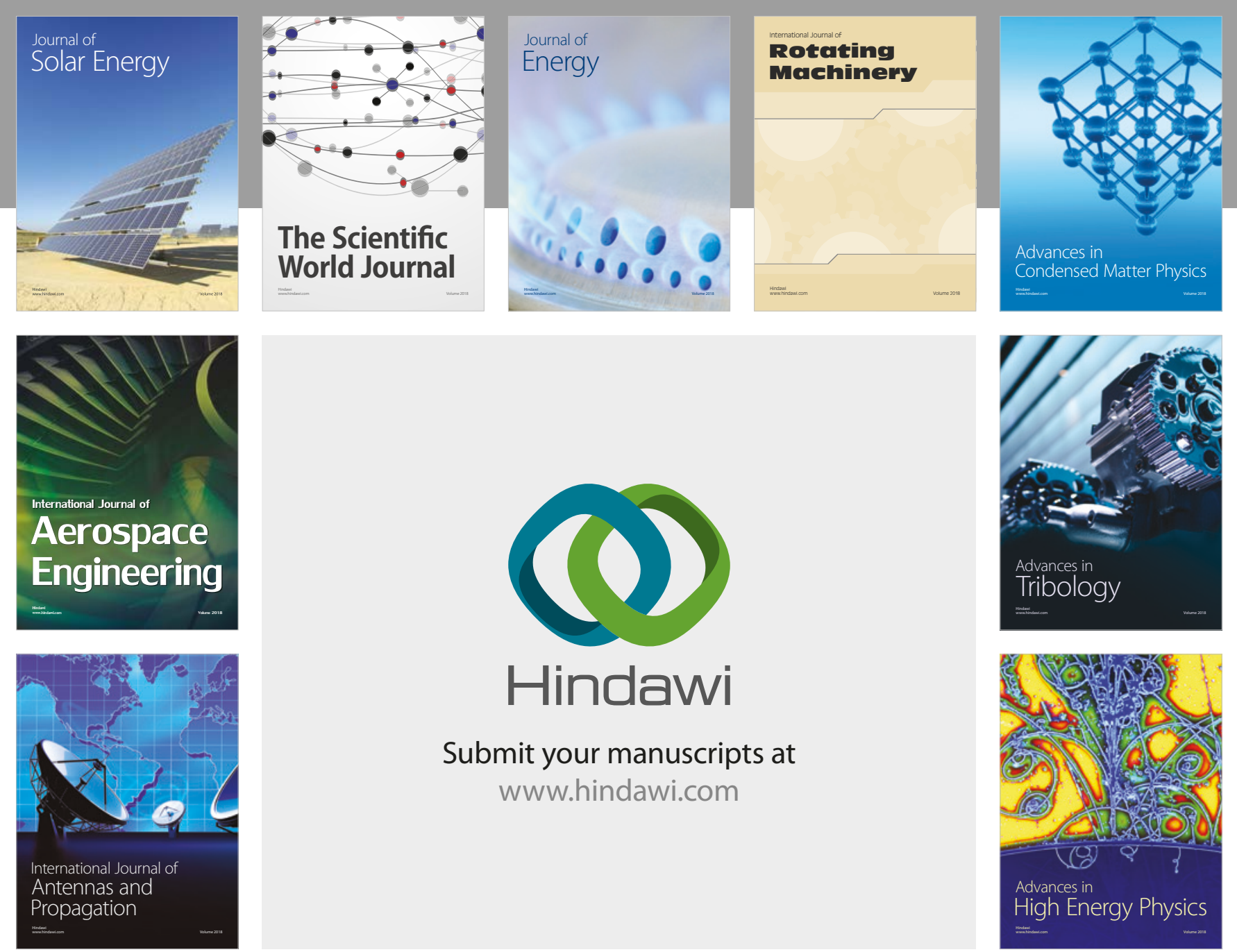

Submit your manuscripts at

www.hindawi.com
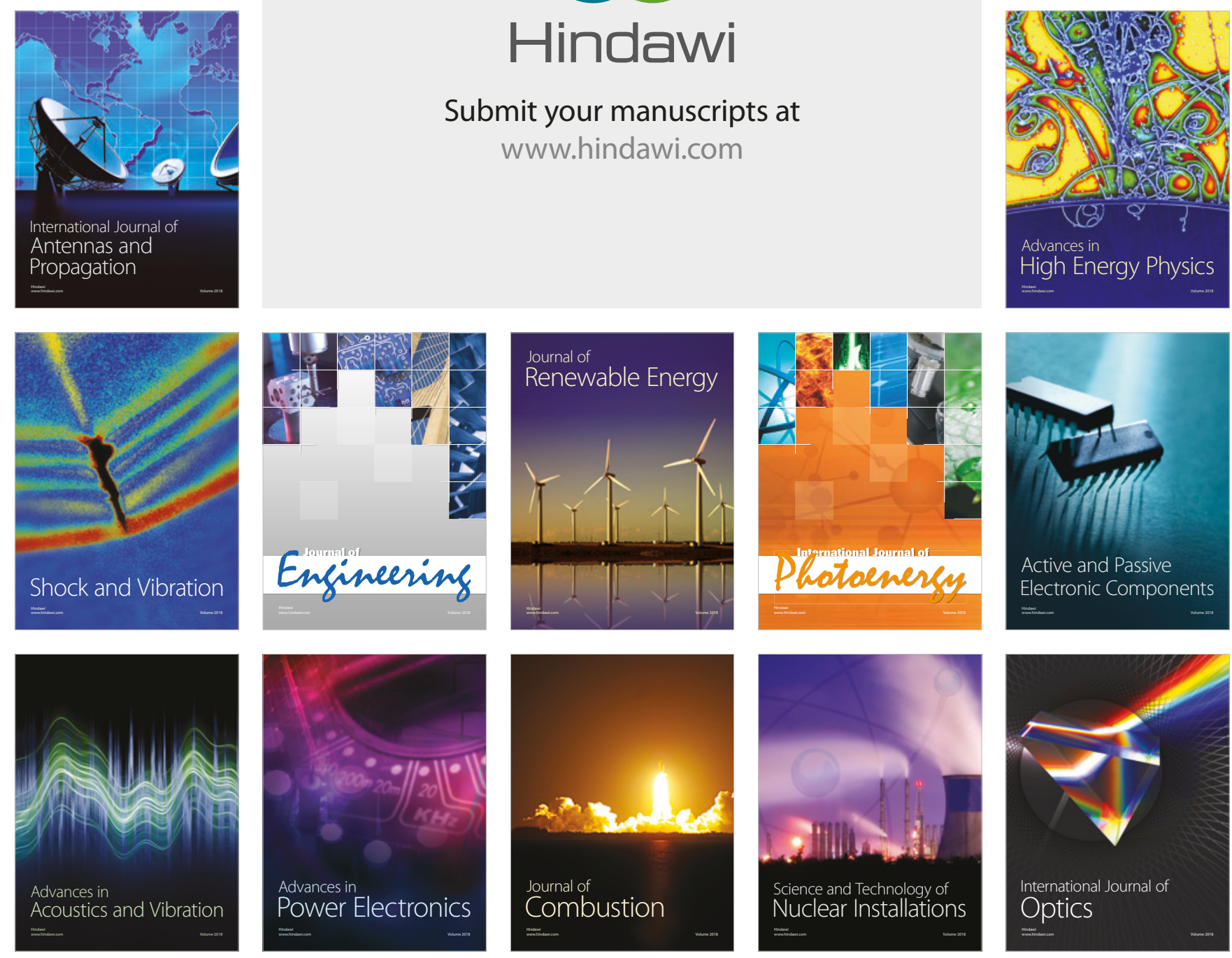